\title{
Comparison of different methods for assessing overall and abdominal obesity among adolescents in Ibadan northwest LGA, Ibadan, Oyo State.
}

\author{
Jasanya OJ, Bello S*, Dairo MD \\ Faculty of Public Health, Department of Epidemiology and Medical Statistics, College of Medicine, University of \\ Ibadan, Nigeria
}

\begin{abstract}
Objectives: The aim of this study was to determine the level of agreement among CDC BMI percentile, WHO BMI Z-scores and IOTF criteria for the assessment of overall obesity and also among the waist circumference, waist to hip ratio, and waist to height ratio criteria for the assessment of abdominal obesity.

Methods: The study involved 602 adolescents identified by multistage sampling method from selected communities. Overall obesity was determined using the CDC BMI percentile, IOTF and WHO BMI $\mathrm{Z}$ score criteria while abdominal obesity was assessed using the WC, WHR, and WHtR. Weight category was divided into underweight, normal, overweight and obesity while the measurement of abdominal adiposity was classified as normal and abdominal obesity. Agreement among the different methods was determined using Kappa coefficient.

Results: The prevalence of overall obesity was $18(3 \%), 17(2.8 \%)$ and $6(1 \%)$ using the CDC BMI percentile, WHO $\mathrm{Z}$ score, and the IOTF criteria respectively while the prevalence of abdominal obesity was $2.3 \%, 9.6 \%$ and $29.1 \%$ using the WC, WHtR and WHR respectively. For the classification of overall obesity, the agreement between the criteria of CDC and WHO BMI $Z$ score was the highest $(K=0.76)$ and the lowest was between IOTF and WHO $(K=0.244)$. For abdominal obesity, the agreement between WC and WHtR $K=0.365$ ) while agreement between WC and WHR gave the lowest $\mathrm{K}=\mathbf{0 . 0 8 8}$.

Conclusion: The highest level of agreement for overall obesity determination obtained was between CDC BMI percentile and WHO BMI Z score while only a moderate agreement was observed between WC and WHtR.
\end{abstract}

Keywords: Adolescents, Body mass index, Overall obesity, Abdominal obesity, Waist circumference.

Abbreviations: CDC: Center for Disease Control and Prevention; WHO: World Health Organization; IOTF: International Obesity Task Force; BMI: Body Mass Index; WC: Waist Circumference Percentile; WHtR: Waist to Height Ratio; WHR: Waist to Hip Ratio; DEXA: Dual Energy X-ray Absorptiometry; MRI: Magnetic Resonance Imaging; LGA: Local Government Area

Accepted on March 02, 2018

\section{Introduction}

Obesity is defined as the accumulation of adipose tissue to a degree where physical and psychosocial health is impaired [1]. It is one of the leading causes of morbidity and mortality in both developed and developing countries and now considered a global epidemic [2] with prevalence increasing in both young and adult populations in as many as 144 countries [3]. The International Obesity Task Force (IOTF) report showed about 10 percent children worldwide is overweight, a total of 155 million children and adolescents are overweight and around 3045 million are classified as obese [4].

Obesity can be assessed using various techniques such as Dual Energy X-ray Absorptiometry (DEXA), underwater weighing and Magnetic Resonance Imaging (MRI) [2]. These methods are the most accurate but are expensive in terms of equipment, utilization, etc thus limiting their use to only clinical research settings. Therefore, because of the lack of simple, accurate methods for assessing body fat directly, anthropometric measures such as skin fold, circumferences and weight and height indices are often used as an alternative for assessing body composition [5]. The BMI measurement is a proven valid method that is cheap and convenient for the assessment of weight status in epidemiological studies and more recently have been recommended for use in children and adolescents [6]. BMI use in the assessment of weight status in young people is more complex, making it essential that age- and sex-specific BMI cut-offs is used to define overweight and obesity [7]. Different methods exist for classifying weight status among adolescents using BMI. For example, the CDC and the WHO uses the age and sex specific cut off points.

New growth charts from the US CDC now include age-and sex-specific BMI reference values for children and adolescents aged 2-20 years [8]. A consensus conference proposed the use of a BMI value above $85^{\text {th }}$ percentile as a screening index for overweight, and a value above the $95^{\text {th }}$ as an index of excess adiposity in adolescents [9]. Others includes the IOTF and the WHO criteria [10]. 
Citation: Jasanya OJ, Bello S, Dairo MD. Comparison of different methods for assessing overall and abdominal obesity among adolescents in Ibadan northwest LGA, Ibadan, Oyo State. J Child Adolesc Health. 2018;2(1):1-5.

Assessment of obesity can also be carried out independent of the BMI by measuring the degree of abdominal adiposity using simple measurements such as waist circumference, waist-to-hip ratio and waist-to-height ratio. Other methods include skin fold thickness, conicity index, etc.

Globally, there is no accepted criterion for classification of overall weight status and abdominal obesity among adolescents [11]. The assessments of weight status among Nigerian children have used several methods and review of studies showed that WHO reference standards were the most commonly used in about $50 \%$ of the studies followed by the IOTF criteria [12]. For abdominal adiposity, the various methods used include skin fold thickness, waist circumference, waist to hip ratio and waist to height ratio and because of the differences in the reference criteria of the above methods, they may produce different estimates of overweight and obesity [13]. Therefore, this study was carried out to compare the prevalence of overall and abdominal obesity among adolescents in Ibadan using different criteria of BMI and waist, hip and height measurements.

\section{Methods}

This cross-sectional observational study was done among adolescents who are resident of Ibadan North West Local Government Area. Ibadan North West is one of the urban LGA in the metropolitan city of Ibadan, Oyo state with an area of 26 $\mathrm{km}^{2}$ and a population of about 152,834 according to the 2006 National Population Census. The LGA inhabitants include Yoruba, Hausa, Ibo and other tribes who engage in trading, farming, artisanship and civil service. Ibadan North West LGA can boost of primary and secondary schools, industries, health and recreational centers.

The study population consisted of 602 adolescents selected using a multistage sampling method. Information on biodata and general characteristics was obtained by intervieweradministered questionnaire. Anthropometry biographical data which included weight, height, and hip circumferences was done by a pre-trained researcher. Body weight was measured using a calibrated measuring scale with a capacity of $130 \mathrm{~kg}$ and $0 \mathrm{~kg}$ precision. The height was measured using a stadiometer with participant standing erect, without shoes, with the back placed against the stadiometer. Body mass index was calculated as weight in $\mathrm{kg}$ divided by height in meters squared. Weight category was divided into underweight, normal, overweight and obese using the Centre for Disease Control and Prevention (CDC) BMI percentile, the International Obesity Task Force (IOTF) and the World Health Organization (WHO) BMI Z score criteria. The WHO BMI Z score classifies individual as values $<-2$ with underweight, normal weight as -2 and 1 , overweight as $Z$ score of $>1$ and $<2$ and obesity as $Z$ score of $\geq 2$ [10]. Using the IOTF criteria, underweight is defined as BMI of $<18.5 \mathrm{Kg} /$ $\mathrm{m}^{2}$, normal weight as BMI of 18.5 to $<25 \mathrm{~kg} / \mathrm{m}^{2}$, overweight as BMI of $\geq 25 \mathrm{~kg} / \mathrm{m}^{2}$ and obesity as BMI of $\geq 30 \mathrm{~kg} / \mathrm{m}^{2}$ while the CDC criteria classified a BMI value above $85^{\text {th }}$ percentile as overweight and a value above $95^{\text {th }}$ as obesity [9]. Waist and hip circumference measurement were taken using an inelastic tape measure at the region slightly above the navel and the widest circumference over the buttock respectively. Abdominal obesity is defined as $\mathrm{WC} \geq 90^{\text {th }}$ percentile for age and sex [11],
WHR $>0.85$ for females and $>0.90$ for males and $\mathrm{WHtR} \geq 0.50$ for age and sex [14].

Ethical approval was obtained from the Oyo State Research Ethical Review Committee, Ministry of Health Secretariat, Ibadan, from the local government authority and the community leaders. All participants were also provided with voluntary written informed consent form of the study to sign after given an opportunity to decide on participation or withdrawal. They were informed of their right to withdraw at any point from the study without any consequences levied against them. Anonymity and confidentiality were assured as no names or contacts of the participants reflected in the questionnaire. Statistical analysis was performed using statistical package for Social sciences version 21. Statistical significance was set $\mathrm{P}<0.05$. The Cohen's Kappa co-efficient was used to analyze the agreement among the various methods for assessing overall and abdominal obesity.

\section{Results}

The respondents' age ranges between 10 and 19 years with mean of 14.88 years and Standard deviation of 2.10 years. Table 1 shows the anthropometric characteristics of respondents. The respondents' BMI ranges between 9.68-32.87 Kg/m² with mean of $19.5 \mathrm{Kg} / \mathrm{m}^{2}$ and standard deviation of $3.19 \mathrm{Kg} / \mathrm{m}^{2}$. Also, there were little or no differences in the mean level of each of the anthropometric characteristics between male and female respondents. Table 2 shows the prevalence of overall obesity. According to the CDC, WHO, and IOTF criteria, overweight and overall obesity is common among the female respondents with the highest prevalence of overweight recorded among females by the WHO BMI Z score. Table 3 shows the prevalence of abdominal obesity. The prevalence of abdominal obesity was highest using WHR (29.1\%). WC and WHtR gave prevalence of $2.3 \%$ and $9.6 \%$ respectively. Table 4 shows the level of agreement among the methods for assessing overall obesity. A substantial agreement was observed with CDC and WHO $(\mathrm{K}=0.756)$ while a moderate and poor agreement were observed with CDC and IOTF $(\mathrm{K}=0.343)$ and with IOTF and WHO $(\mathrm{K}=0.244)$ respectively. Table 5 shows the level of agreement among the methods for assessing abdominal obesity. A moderate agreement was observed with WC and WHtR $(\mathrm{K}=0.365)$. A fair agreement was recorded with WHtR and WHR $(\mathrm{K}=0.282)$ while WC and WHR $(\mathrm{K}=0.088)$ gave a very poor agreement. Table 6 shows the distribution of respondents based on overall obesity measured by WHO BMI Z s-core and central obesity using various methods. Fourteen $(100 \%)$ of respondents with abdominal obesity using WC were also overweight or obese using the WHO BMI z-score compared to $60 \%$ using WHtR and $20 \%$ using WHR.

\section{Discussion}

This study was a community-based and out of the total respondents, $335(55.6 \%)$ were females. The mean age of the study population was 14.28 years with a standard deviation of 2.10 years. About $39 \%$ of respondents were in the early adolescence while the remaining 367 (61\%) were late adolescents. This study showed a high prevalence of excess weight (overweight and obesity) in a sample of 602 adolescents and these values varied according to the criteria used, 5.5\% by IOTF, $8.0 \%$ by CDC and $10.3 \%$ using the WHO criteria. 
Table 1. Anthropometric characteristic of the respondents.

\begin{tabular}{|c|c|c|}
\hline Variables & Males (N) s.d & Females (N) s.d \\
\hline Height (in meters) & $1.62(0.12)$ & $1.58(0.09)$ \\
\hline Weight (in kg) & $51.53(11.28)$ & $48.67(10.70)$ \\
\hline Waist circumference $(\mathrm{cm})$ & $68.84(7.73)$ & $70.15(7.94)$ \\
\hline Hip circumference $(\mathrm{cm})$ & $81.11(9.68)$ & $84.12(9.95)$ \\
\hline Body Mass Index $\left(\mathrm{kg} / \mathrm{m}^{2}\right)$ & $19.46(2.85)$ & $19.50(3.44)$ \\
\hline Waist to height ratio & $0.43(0.04)$ & $0.45(0.05)$ \\
\hline Waist to hip ratio & $0.85(0.05)$ & $0.04)$ \\
\hline
\end{tabular}

Table 2. Prevalence of weight status according to BMI percentile, IOTF, and WHO.

\begin{tabular}{|c|c|c|c|c|c|c|}
\hline \multirow{2}{*}{ Variables } & \multicolumn{2}{|c|}{ BMI percentile } & \multicolumn{2}{|c|}{ BMI Z score } & \multicolumn{2}{|c|}{ IOTF BMI } \\
\hline & Male (\%) & Female (\%) & Male (\%) & Female (\%) & Male (\%) & Female (\%) \\
\hline Underweight & $34(12.7)$ & $32(9.6)$ & $18(6.7)$ & $26(7.8)$ & $94(35.2)$ & $144(43.0)$ \\
\hline Normal & $216(80.9)$ & $272(81.2)$ & $227(85.0)$ & 269 (80.3) & $165(61.8)$ & $166(49.6)$ \\
\hline Overweight & $11(4.1)$ & $19(5.7)$ & $17(6.4)$ & $28(8.4)$ & $7(2.6)$ & $20(6.0)$ \\
\hline Obesity & $6(2.2)$ & $12(3.6)$ & $5(1.9)$ & $12(3.6)$ & $1(0.4)$ & $5(1.5)$ \\
\hline Total & $267(100)$ & $335(100)$ & $267(100)$ & $335(100)$ & $267(100)$ & $335(100)$ \\
\hline
\end{tabular}

Table 3. Prevalence of central obesity according to WC, WHtR, and WHR.

\begin{tabular}{|c|c|c|c|c|c|c|}
\hline \multirow{2}{*}{ Category } & \multicolumn{3}{|c|}{ WC } & \multicolumn{2}{|c|}{ WHtR } & \multicolumn{2}{c|}{ WHR } \\
\cline { 2 - 7 } & Male\% & Female\% & Male\% & Female\% & Male\% & Female\% \\
\hline Normal & $264(98.9)$ & $324(96.7)$ & $253(94.8)$ & $291(86.9)$ & $234(87.6)$ & $193(57.6)$ \\
\hline Obese & $3(1.1)$ & $11(3.3)$ & $14(5.2)$ & $44(13.1)$ & $33(12.4)$ & $142(42.4)$ \\
\hline Total & $267(100)$ & $335(100)$ & $267(100)$ & $335(100)$ & $267(100)$ & $335(100)$ \\
\hline
\end{tabular}

Table 4. Kappa co-efficient for the classification of weight status according to the three criteria for classification of BMI.

\begin{tabular}{|c|c|c|c|}
\hline Gender & CDC vs IOTF & CDC vs WHO & IOTF vs WHO \\
\hline Male & 0.390 & 0.701 & 0.756 \\
\hline Female & 0.314 & 0.233 & 0.233 \\
\hline Total & 0.343 & 0.795 & 0.244 \\
\hline
\end{tabular}

Table 5. Kappa co-efficient for the classification of central obesity according to the three criteria for classification of WC, WHtR and WHR.

\begin{tabular}{|c|c|c|c|}
\hline Gender & WC vs WHtR & WC vs WHR & WHtR vs WHR \\
\hline Male & 0.341 & 0.367 & 0.365 \\
\hline Female & 0.092 & 0.074 & 0.088 \\
\hline Total & 0.288 & 0.247 & 0.282 \\
\hline
\end{tabular}

Table 6. Distribution of respondents based on overall obesity measured by WHO BMI Z score and central obesity using various methods.

\begin{tabular}{|c|c|c|c|c|c|c|c|}
\hline \multirow{2}{*}{ WHO BMI } & \multirow{2}{*}{ N \% } & \multicolumn{2}{|c|}{ WC percentile } & \multicolumn{2}{|c|}{ WHtR } & \multicolumn{2}{|c|}{ WHR } \\
\cline { 2 - 7 } & & Normal \% & Obese \% & Normal \% & Obese \% & Normal \% & Obese \% \\
\hline Underweight & $44(7.3)$ & $44(100.0)$ & $0(0.0)$ & $42(95.5)$ & $2(4.5)$ & $25(56.8)$ & $19(43.2)$ \\
\hline Normal & $496(82.4)$ & $496(100.0)$ & $0(0.0)$ & $475(95.8)$ & $21(4.2)$ & $375(75.6)$ & $121(24.4)$ \\
\hline Overweight & $45(7.5)$ & $38(84.4)$ & $7(15.6)$ & $23(51.1)$ & $22(48.9)$ & $23(51.1)$ & $22(48.9)$ \\
\hline Obesity & $17(2.8)$ & $10(58.8)$ & $7(41.2)$ & $4(23.5)$ & $13(76.5)$ & $4(23.5)$ & $13(76.5)$ \\
\hline Total & $602(100)$ & $588(97.7)$ & $14(2.3)$ & $544(90.4)$ & $58(9.6)$ & $427(70.9)$ & $175(29.1)$ \\
\hline
\end{tabular}

The cutoffs WHO criteria produced the highest estimates of the prevalence of excess weight and the IOTF the lowest. This finding was consistent with a Portuguese study where a higher prevalence of excess weight was also recorded using the WHO and lowest by the IOTF criteria [15]. Similar findings were also reported by Sadinha with WHO BMI Z score giving the highest prevalence of excessive weight [14].

In this study, more females $(11.9 \%)$ were overweight or obese compared to males $(8.2 \%)$. This is consistent with a Port Harcourt study [16] which showed a higher prevalence among females $(2.4 \%)$ than males $(0.42 \%)$ and in Ibadan [17] where higher prevalence was also observed among females compared to their male counterparts whereas a contrasting findings were reported in a Greece study [18] and Chinese study [19]. The observed higher prevalence among the female adolescents may be due to the fact that during adolescence, female tends to lay down more fat which might be responsible for the visibility of rotundity in them. Testosterone secretion inhibits the process among male adolescents [20].

The prevalence of abdominal obesity as determined using $\mathrm{WC}$, WHtR, and WHR was 2.3\%, 9.6\%, and 29.1\% respectively. Similar to overall obesity, the prevalence was also higher among females compared to male. The WHR cut off gave the highest prevalence $(42.4 \%)$ followed by WHtR (13.1\%) and the WC the lowest $(3.3 \%)$. Similar findings were reported in a Port Harcourt study among adolescent girls with WHR giving 
Citation: Jasanya OJ, Bello S, Dairo MD. Comparison of different methods for assessing overall and abdominal obesity among adolescents in Ibadan northwest LGA, Ibadan, Oyo State. J Child Adolesc Health. 2018;2(1):1-5.

the highest prevalence (47.81\%) and WC (1.5\%) lowest [21] while an Iranian study among adolescent girls reported highest prevalence of abdominal obesity with WC (13.2\%) [22], a contrast to the finding of this study. The contrasting report may be as result of varied criteria used to define the references. Age differences and population studied might also account for the inconsistencies.

Using Cohen's Kappa coefficient, the agreement between the various criteria for assessing overall and central obesity varied with age and sex with the highest agreement observed among female in their early adolescence. For the overall obesity, the agreement between the WHO Z score and the BMI according to the IOTF was fair $(K=0.233)$ while the agreement between the WHO Z score and the BMI percentile was strong $(\mathrm{K}=0.756)$. Similar to this finding was the report by Twells and New Hook [23] where the highest level of agreement was also between the CDC BMI percentile and WHO BMI $z$-score $(\mathrm{K}=0.84)$ and contrary to the above findings was a Portuguese study that reported the highest agreement between the BMI percentile and the IOTF criteria $(\mathrm{k}=0.79-0.88)$ [15]. This study also showed a poor to fair agreements between the alternative criteria for assessing abdominal obesity among adolescents. The highest agreements recorded was between the WC percentile and WHtR $(\mathrm{K}=0.365)$ followed by between WHtR and WHR $(\mathrm{k}=0.282)$ while a very agreement poor agreement was observed with WC percentile and WHtR $(\mathrm{k}=0.088)$.

This study showed that larger percentage of adolescents who were abdominally obese using the Waist Circumference and Waist to Height Ratio criteria was also found to have excessive overall weight status measured using WHO BMI z-score indicating a strong correlation between the measures. Consistence with other studies [20], [24] waist to hip ratio, a measure of abdominal obesity correlates poorly with measures of overall weight status among adolescents.

\section{Conclusion}

In conclusion, the prevalence of both overall and central obesity is relatively higher among the female adolescents compared to their counterparts. The CDC BMI percentile and the WHO BMI $\mathrm{Z}$ score have the strongest agreements while only a moderate agreement was observed between waist circumference percentile and waist to height ratio.

Further studies are suggested to use a large sample size in order to define the best methods for accessing weight status and adiposity during adolescence.

\section{Limitation}

The drawback of the study was the inadequate access to primary data because due the difficulty in obtaining consent from the adolescent parents to participate in the study. Also, the anthropometric assessment of the adolescents took a lot time limiting the number of adolescents who were willing to participate.

\section{Strength}

The major strength of this study is that the findings can be generalized since the sample procedure was well designed and the study sample is a representative of the study population.

\section{Acknowledgement}

We are indebted to all participants in this study for their cooperation. Also, we thank Dr M.D. Dairo, Dr S. Bello, Miss O.O. Daodu and Miss Badru M. for their valuable support during the survey.

\section{Conflict of Interest}

The authors declare: No conflict of interest.

\section{Funding}

No Funding.

\section{References}

1. James PT. Obesity: The worldwide epidemic. Clin Dermatol. 2004;22:276-80.

2. World Health Organization. Physical status: The use and interpretation of anthropometry. Report of a WHO Expert committee. WHO Technical Report Series No. 854. WHO, Geneva, Switzerland. 1995.

3. Onis M, Blossner M, Borghi E. Global prevalence and trends of overweight and obesity among preschool children. Am J Clin Nutr. 2010;92:1257-64.

4. IOTF. Press Release: IOTF demands action on childhood obesity crisis. International Obesity Task Force;2007.

5. Onis M, Habicht JP. Anthropometric reference data for international use: recommendations from a World Health Organization Expert Committee. Am J Clin Nutr. 1996;64:650-8.

6. Widhalm K, Schonegger K, Huemer C, et al. Does the BMI reflect body fat in obese children and adolescents? A study using the TOBEC method. Int J Obes Relat Metab Disord. 2001;25:279-85.

7. Field AE, Laird N, Steinberg E, et al. Which metric of relative weight best captures body fatness in children? Obesity Res. 2003;11:1345-52.

8. Kuczmarski RJ, Ogden CL, Grummer-Strawn LM, et al. CDC growth charts: United States. Adv Data. 2000;8:1-27.

9. Must A, Dallal GE, Dietz WH. Reference data for obesity: 85th and 95th percentiles of body mass index (wt/ht2) and triceps skin fold thickness. Am J Clin Nutr. 1991;53:839-46.

10. Onis M, Blossner M. Prevalence and trends of overweight among preschool children in developing countries. Am J Clin Nutr 2000;72:1032-9.

11. Centers for Disease Control and Prevention. About BMI for Children and Teens.

12. Ejike CE. Child and adolescent obesity in Nigeria: A narrative review of prevalence data from three decades (1983-2013). J Obes Metab Res. 2014;1:171-9.

13. Kain J, Uauy R, Vio F, et al. Trends in overweight and obesity prevalence in Chilean children: comparison of three definitions. Eur J Clin Nutr. 2002;56:200-4.

14. Sardinha L, Santos R, Vale M, et al. Waist circumference 
percentiles for Portuguese children and adolescents aged 10 to 18 year. Eur J Pediatr. 2012;171:499-505.

15. Minghelli B, Nunez C, Oliviera R. Body mass index and waist circumference to define thinness, overweight and obesity in Portuguese adolescents: comparison between CDC, IOTF and WHO references. Pediatr Endocrinol Rev. 2014; $12: 35-41$.

16. Buowari Y. Obesity among students attending a tertiary institution in Nigeria. Internet J Trop Medicine. 2009;7:102-9.

17. Omigbodun OO, Adediran KI, Akinyemi JO, et al. Gender and rural-urban differences in the nutritional status of the in-school adolescent in western Nigeria. J Biosoc Sci. 2010;42:65-76.

18. Patsopoulou A, Tsimtsiou Z, Katsioulis A, et al. Prevalence and risk factors of overweight and obesity among adolescents and their parents in Central Greece (FETA Project). Int J Environ Res Public Health. 2015;13:1-10.

19. Guo X, Zheng L, Li Y, et al. Prevalence and risk factors of being overweight or obese among children and adolescents in northeast China. Pediatr Res. 2013;74:443-9.

20. Vizmanos B, Martı'-Henneberg C. Puberty begins with a characteristic subcutaneous body fat mass in each sex. Eur J Clin Nutr. 2000;54:203-8.

21. Jaja T, Alex HB. Prevalence of overall and central obesity among adolescent girls in Port Harcourt: A comparison of different methods. Niger J Paediatr. 2016;43:209-14.

22. Rafraf M, Mohamadi E, Gargari BP. Prevalence of overall and abdominal obesity among adolescent high school girls in Tabriz, Iran. Int Med J Malaysia. 2013;12:27-32.

23. Twells L, Newhook L. Obesity prevalence estimates in a Canadian regional population of preschool children using variants growth references. BMC Pediatr. 2011;11:21.

24. Sabageh AO, Ojofeitimi EO. Prevalence of obesity among adolescents in Ile-Ife, Osun State, Nigeria using body mass index and waist to hip ratio: A comparative study. Niger Med J. 2013;54:153-6.

\section{*Correspondence to:}

Dr S. Bello

Faculty of Public Health

Department of Epidemiology and Medical Statistics

College of Medicine

University of Ibadan

Nigeria

E-mail: drsegunbello@yahoo.com 